\title{
ON THE LOCAL MONODROMY OF A VARIATION OF HODGE STRUCTURE
}

\author{
BY EDUARDO CATTANI ${ }^{1}$ AND AROLDO KAPLAN ${ }^{1}$
}

Associated to a variation of polarized Hodge structure there is a period mapping $\psi: S \rightarrow \Gamma \bigvee$, where $S$ is the parameter space and $\Gamma \bigvee D$ denotes the corresponding modular variety of polarized Hodge structures (the primary example to keep in mind is that arising from a family of smooth projective varieties parametrized by $S$ ) [3] , [4]. The local study of the singularities of $\psi$ ([5]) reduces to the case when $S=\left(\Delta^{*}\right)^{l} \times \Delta^{m}$, a product of punctured disks and disks.

Given a lifting $\widetilde{\psi}: U^{l} \times \Delta^{m} \rightarrow D(U=$ upper half-plane) of $\psi$ to the universal covering of $S$ there are monodromy transformations $\gamma_{1}, \ldots, \gamma_{l} \in \Gamma$ such that

$$
\begin{aligned}
\widetilde{\Psi}\left(z_{1}, \ldots, z_{i}+1, \ldots, z_{l} ; w_{1}, \ldots, w_{m}\right) & \\
& =\gamma_{i} \widetilde{\psi}\left(z_{1}, \ldots, z_{i}, \ldots, z_{l} ; w_{1}, \ldots, w_{m}\right) .
\end{aligned}
$$

These $\gamma_{i}$ 's, which are quasi-unipotent automorphisms of the $\mathrm{C}$-vector space $H$ underlying the variation, provide important invariants of the singularities of $\psi$. In particular, in the single variable case $(l=1, m=0)$ a central role is played by the monodromy weight filtration $W_{*}=W_{*}(N)$ of the nilpotent transformation $N=\log \gamma_{u}$, where $\gamma_{u}$ is the unipotent part of the monodromy $\gamma$. We recall [5] that, if $k$ is the weight of the Hodge structures, $N^{k+1}=0$ and the filtration (0) $\subseteq W_{0} \subseteq \cdots \subseteq W_{2 k}=H$ is uniquely characterized by the conditions $N W_{j} \subseteq$ $W_{j-2}$ and $N^{j}: W_{k+j} / W_{k+j-1} \rightarrow W_{k-j} / W_{k-j-1}$ is an isomorphism.

The results announced here concern the monodromy weight filtrations arising in the several variables case. The main statements-Theorems 1 and 2were conjectured by P. Deligne [2] (cf. Conjecture 1.9.6, as well as Theorem 1.9.2 for the special geometric case). For structures of weight two they are contained in [1].

THEOREM 1. Let $\gamma_{1}, \ldots, \gamma_{l}$ be monodromy transformations of a period mapping $\psi:\left(\Delta^{*}\right)^{l} \times(\Delta)^{m} \rightarrow \Gamma \bigvee$ and $N_{i}$ the logarithm of the unipotent part

Received by the editors August 14, 1980.

1980 Mathematics Subject Classification. Primary 14C30, 32G20; Secondary 22E40, $32 \mathrm{M} 10$.

1 Research supported in part by an NSF Grant.

Some of the work in this paper was done while the first author was visiting Leiden University under the auspicies of the Netherland Organization for the Advancement of Pure Research (Z.W.O.), and the Institute des Hautes Études Scientifiques. 
of $\gamma_{i}$. Then all the elements in the open cone of commuting nilpotent endomorphisms

$$
\sigma=\left\{\sum_{i=1}^{l} \lambda_{i} N_{i} ; \lambda_{i} \in \mathbf{R}, \lambda_{i}>0\right\}
$$

define the same monodromy weight filtration (to be denoted by $W_{*}(\sigma)$ ).

According to Schmid's Nilpotent Orbit Theorem [5] there exists a limiting Hodge filtration $F^{*}$ (depending on $w_{1}, \ldots, w_{m}$ ) such that the lifting $\widetilde{\psi}$ may be approximated for $\operatorname{Im} z_{j}>>0$ by the orbit $\exp \left(\Sigma_{j} z_{j} N_{j}\right) \cdot F^{*}$. Moreover in the case of a single $N$ and as a consequence of the $S L_{2}$-orbit theorem, $\left(W_{*}(N), F^{*}\right)$ defines a polarized mixed Hodge structure, i.e. the Hodge filtration $F^{*}$ defines Hodge structures on the graded quotients $\operatorname{Gr}_{j}(N)=W_{j}(N) / W_{j-1}(N)$ which are suitably polarized (cf. Theorem 6.16 in [5] for the precise statement, as well as [6]). When this is combined with Theorem 1 one obtains that every point in the approximating orbit defines a mixed Hodge structure relative to the filtration $W_{*}(\sigma)$.

A further consequence of Theorem 1 is the existence of a Hodge filtration $F_{0}^{*}$ such that $\left(W_{*}(\sigma), F_{0}^{*}\right)$ is a mixed Hodge structure split over $\mathbf{R}$ and the orbit $\exp \left(\Sigma_{j} z_{j} N_{j}\right) \cdot F_{0}^{*}$ lies in $D$ for $\operatorname{Im} z_{j}>0$. For a single monodromy transformation these "split" nilpotent orbits correspond to $S L_{2}$-orbits in the sense of [5]. Thus they could be expected to play a role in extending the $S L_{2}$-orbit theorem to the case of period mappings of several variables.

The second theorem relates the weight filtration $W_{*}(\sigma)$ to those associated to the faces of the cone $\sigma$. In order to make this statement precise let us consider two commuting nilpotent transformations $N$ and $N^{\prime}$. Since $N^{\prime}$ preserves $W_{*}(N)$, it induces nilpotent endomorphisms in each of the graded quotients $\mathrm{Gr}_{j}(N)$. Suppose that the monodromy weight filtrations defined by $N^{\prime}$ in the various $\operatorname{Gr}_{j}(N)$ 's are all projections of a single filtration $W_{*}$ of the total space with the property that $N^{\prime} W_{j} \subseteq W_{j-2}$ (such a $W_{*}$ need not exist for an arbitrary commuting pair $N, N^{\prime}$, but if it does, it is unique [2]). Then, following Deligne, we call such $W_{*}$ the monodromy weight filtration of the pair $\left(N^{\prime}, W_{*}(N)\right)$.

THEOREM 2. Let $N, N^{\prime}$ be any two elements in the closure of $\sigma$ not belonging to the same proper face of this cone. Then $W_{*}(\sigma)$ is the monodromy weight filtration of the pair $\left(N^{\prime}, W_{*}(N)\right)$.

We end with a bare bones sketch of the proof of Theorem 1. For elementary reasons there exists a Zariski-open subcone $\sigma^{\prime}$ of $\sigma$ where the map $N \rightarrow$ $W_{*}(N)$ takes values in a fixed flag manifold. Then the existence of a Hodge filtration $F^{*}$ such that $\left(W_{*}(N), F^{*}\right)$ is a polarized mixed Hodge structure for all $N \in \sigma$ is seen to imply the vanishing of the differential of that map. Hence the filtration $W_{*}(N)$ must be constant on each connected component of $\sigma^{\prime}$. 
For any such component $\sigma_{0}^{\prime}$ it is now possible to construct a new Hodge filtration $F_{0}^{*}$ such that $\left(W_{*}\left(\sigma_{0}^{\prime}\right), F_{0}^{*}\right)$ is a mixed Hodge structure split over $\mathbf{R}$ and such that $\exp z N \cdot F_{0}^{*} \in D$ for $N \in \sigma_{0}^{\prime}$ and $\operatorname{Im} z>0$. This, combined with Lemma 1 in [1], guarantees in turn that $\left(W_{*}(N), F_{0}^{*}\right)$ is a mixed Hodge structure even for an $N$ in the closure of $\sigma_{0}^{\prime}$ in $\sigma$. The conclusion that $W_{*}(N)=W_{*}\left(\sigma_{0}^{\prime}\right)$ for any such $N$ and, hence, that of Theorem 1, follows from the Proposition below which, together with the reduction to the $\mathbf{R}$-split case, also underlies the proof of Theorem 2 .

Proposition. Let $\left(W_{*}, F^{*}\right)$ be a mixed Hodge structure split over $\mathbf{R}$ and $N$ a $(-1,-1)-m o r p h i s m$ of it. Then $\left(W_{*}(N), F^{*}\right)$ is a mixed Hodge structure if and only if $W_{*}(N)=W_{*}$.

Detailed proofs will appear elsewhere. We wish to thank Pierre Deligne for his generous advice and encouragement during the preparation of this work.

\section{REFERENCES}

1. E. Cattani and A. Kaplan, The monodromy weight filtration for a several variables degeneration of Hodge structures of weight two, Invent. Math. 52 (1979), 131-142.

2. P. Deligne, La conjecture de Weil. II, Publ. Math. Inst. Hautes Études. Sci. (to appear).

3. P. Griffiths, Periods of integrals on algebraic manifolds. I, II, Amer. J. Math. 90 (1968), 568-626; 805-865.

4. Periods of integrals on algebraic manifolds. III, Publ. Math. Inst. Hautes Études Sci. 38 (1970), 125-180.

5. W. Schmid, Variation of Hodge structure: the singularities of the period mapping, Invent. Math. 22 (1973), 211-319.

6. J. Steenbrink, Limits of Hodge structures, Invent. Math. 31 (1976), 229-257

DEPARTMENT OF MATHEMATICS AND STATISTICS, UNIVERSITY OF MASSACHUSETTS, AMHERST, MASSACHUSETTS 